\title{
Separation of Normal and Premalignant Cervical Epithelial Cells Using Confocal Light Absorption and Scattering Spectroscopic Microscopy Ex Vivo
}

\author{
Ling Yang, ${ }^{1,2}$ Wen-Tao Liu, ${ }^{3}$ Hao Wu, ${ }^{4}$ Cheng Wang, ${ }^{5}$ Bo Ping, ${ }^{1,2}$ and Da-Ren Shi ${ }^{1,2}$ \\ ${ }^{1}$ Department of Pathology, Fudan University Shanghai Cancer Center, Shanghai 200032, China \\ ${ }^{2}$ Department of Oncology, Shanghai Medical College, Fudan University, Shanghai 200032, China \\ ${ }^{3}$ Department of Surgery, Ruijin Hospital, Shanghai Institute of Digestive Surgery, Shanghai Jiao Tong University, \\ 200025 Shanghai, China \\ ${ }^{4}$ Department of Obstetrics and Gynecology, Shanghai First People's Hospital, Shanghai Jiao Tong University, 200080 Shanghai, China \\ ${ }^{5}$ Institute of Medical Optics and Optometry, University of Shanghai for Science and Technology, 200093 Shanghai, China
}

Correspondence should be addressed to Da-Ren Shi, shidaren@yahoo.com.cn

Received 16 April 2011; Accepted 25 July 2011

Academic Editor: Manoor Prakash Hande

Copyright () 2011 Ling Yang et al. This is an open access article distributed under the Creative Commons Attribution License, which permits unrestricted use, distribution, and reproduction in any medium, provided the original work is properly cited.

Confocal light absorption and scattering spectroscopic (CLASS) microscopy can detect changes in biochemicals and the morphology of cells. It is therefore used to detect high-grade cervical squamous intraepithelial lesion (HSIL) cells in the diagnosis of premalignant cervical lesions. Forty cervical samples from women with abnormal Pap smear test results were collected, and twenty cases were diagnosed as HSIL; the rest were normal or low-grade cervical squamous intraepithelial lesion (LSIL). The enlarged and condensed nuclei of HSIL cells as viewed under CLASS microscopy were much brighter and bigger than those of nonHSIL cells. Cytological elastic scattered light data was then collected at wavelengths between 400 and $1000 \mathrm{~nm}$. Between $600 \mathrm{~nm}$ to $800 \mathrm{~nm}$, the relative elastic scattered light intensity of HSIL cells was higher than that of the non-HSIL. Relative intensity peaks occurred at $700 \mathrm{~nm}$ and $800 \mathrm{~nm}$. CLASS sensitivity and specificity results for HSIL and non-HSIL compared to cytology diagnoses were $80 \%$ and $90 \%$, respectively. This study demonstrated that CLASS microscopy could effectively detect cervical precancerous lesions. Further study will verify this conclusion before the method is used in clinic for early detection of cervical cancer.

\section{Introduction}

Cervical cancer is a very common malignancy in women. The National Cervical Cancer Coalition (NCCC) estimates that worldwide, 473,000 new cases of cervical cancer are diagnosed and 253,500 patients die of this disease annually. This makes cervical cancer the twelfth most common cancer and the fifth cause of cancer-related deaths in the world [1]. Approximately $85 \%$ of cervical cancers occur in developing countries. Human papillomavirus (HPV) infection is the most important risk factor for the development of cervical cancer [2]. Treatment of cervical cancer consists of surgery at the early stages and chemotherapy and radiation therapy for advanced disease.

Prognosis of cervical cancer depends on the cancer stage. If diagnosed early, the 5-year survival rate of the patient is more than $90 \%$. Therefore, early detection is currently the most effective means to improve survival of patients. The Papanicolaou (Pap smear screening) test followed by colposcopy is the most frequently used means to detect precancerous and cancerous changes in the cervix. However, the current most practical Pap smear screening plus cytology test has a low sensitivity, especially in discriminating highgrade squamous intraepithelial lesions (HSILs) from other abnormalities and normal tissues [3]. In routine clinical practice, inaccurate Pap smear results and colposcopic impressions often lead to overtreatment of patients [4]. Thus, development of a more sensitive and specific method to detect cervical cancer early and accurately is urgently needed.

For several past decades, we have witnessed the rapid development and use of optical methods to investigate changes in cell behaviors (such as migration and intracellular 
protein movement) and for disease diagnosis; optical techniques enable the detection of changes in biochemical and morphological features that are concurrent with precancerous conditions [5]. The advantage of optical techniques is that in living cells visible light does no harm or is relatively benign. Optical techniques such as confocal reflectance microscopy, optical coherence tomography, light-scattering spectroscopy, and elastic scattering spectroscopy are able to distinguish and identify intrinsic optical properties of tissues.

During the transition from normal to cancerous cells, biochemical, molecular, and morphologic alterations may lead to changes in the cells' optical properties. Indeed, recent studies have shown that elastic light-scattering spectroscopy may permit the earliest detection of carcinogenesis. Lightscattering spectroscopy (LSS) is an optical technique in which quantitative information on cell organelle morphology is extracted via measurement of the spectra and angular distribution of backscattered light. Therefore, LSS could detect precancerous and early cancerous changes in tissues $[6]$.

In this study, we investigated the novel technique confocal light absorption and scattering spectroscopic (CLASS) microscopy to detect the light scattering differences in liquidbased cervical cell samples. CLASS combines LSS with confocal microscopy for early cancer detection [7, 8]. CLASS provides not only size information but also the biochemical and physical properties of the cells. It images tissue at greater depth than conventional confocal microscopy and provides enhanced image contrast. In addition, by utilizing backscattered light to detect subcellular structures, CLASS can detect changes in living cells without introducing exogenous labels [5].

In the present study, we demonstrate that CLASS microscopy could distinguish between populations of normal, low-grade squamous intraepithelial lesion (LSIL), and HSIL cells ex vivo, and therefore could be developed for clinical use in the accurate and precise diagnosis of Pap smear samples.

\section{Materials and Methods}

2.1. Cell Samples. Forty women aged twenty and older who had a history of abnormal cervical cytology were enrolled in this study at the Department of Pathology, Cancer Hospital of Fudan University, between 2009 and 2010. Our institutional review board approved the research protocol for this work. A written informed consent form was obtained from each subject. Of these 40 patients, 20 had cytologically confirmed HSIL and 20 were non-HSIL. All cytological samples were free of blood clot and stored at $4^{\circ} \mathrm{C}$ for experimental procedures within $24 \mathrm{~h}$.

2.2. Sample Preparation for CLASS Analysis. To perform CLASS analysis, the cytological specimens were first put into ThinPrep preservation solution containing deionized water, $0.1 \%$ Polysorbate 20 (Tween-20) detergent, and 54\% ethanol. The pathologist then mapped a region of interest, designating the region that contained the worst cell lesions.
TABLE 1: CLASS data compared to cytology diagnosis.

\begin{tabular}{lccc}
\hline $\begin{array}{l}\text { Relative } \\
\text { intensity }\end{array}$ & $\begin{array}{c}\text { HSIL }>\text { Non-HSIL } \\
\text { (CLASS) }\end{array}$ & $\begin{array}{c}\text { HSIL }<\text { Non-HSIL } \\
\text { (CLASS) }\end{array}$ & $P$ value \\
\hline HSIL & 16 & 4 & $P>0.05$ \\
Non-HSIL & 2 & 18 & \\
\hline
\end{tabular}

Cells were suspended at $10^{8}$ cells $/ \mathrm{mL}$ to avoid overlapping nuclei in the analysis. Cells in saline were used as the control.

2.3. Spectroscopy. Cells in Thinprep or in saline (as the control) were kept in an open volume container or dipped onto glass slides. The container was black on the inside in order to minimize edge effects. Measurements were made with a fiber optic probe placed on the surface of the cell suspension.

To perform the CLASS analysis, we used a prototype CLASS microscope as described in a previous report [5]. After the optical signal was collected by the CLASS receiver, the data was analyzed using spectral data acquisition BWspec software (B\&W, Newark, DE, USA). Before every experiment, the light base was adjusted to minimize the signal-tonoise ratio.

2.4. Data Analysis. The data was first normalized to the saline control samples and then comparisons were made between the HSIL and non-HSIL samples. SPSS 13.0 statistical software was used to analyze the summarized data. Sensitivity and specificity were calculated by percentage of cases correctly identified by CLASS as compared to those confirmed by cytology, and then presented as mean \pm standard deviation (SD). Student's $t$-test was performed to generate a probability $P$ value. $P<0.05$ was considered statistically significant.

\section{Results}

3.1. Study Population. From patients with abnormal Pap smear cytology results, we collected 20 cervical cytology samples of HSIL, and 20 of non-HSIL. In the HSIL samples, the pathology report showed 6 cases with lesions of cervical intraepithelial neoplasia (CIN) grade II, and 14 cases with lesions of CIN grade III. In the non-HSIL samples, 8 cases showed normal cytology, and 12 cases were positive for LSIL (Table 1).

3.2. Comparison of Microscopic and CLASS Photographs. One representative image each of normal, LSIL, and HSIL cells is shown in Figure 1 (left to right, resp.). We chose cell samples from 4, 7, and 9 confirmed diagnosed normal, LSIL, and HSIL cytology specimens, respectively, for CLASS analysis.

Using CLASS microscopy, these cells show a unclear edge, but bright nuclei. In the CLASS method of cell imaging, enlarged nuclei become brighter (Figure 2). Thus, nuclei that have changed in size and condensation are detected by LSS and are early indications of malignant transformation. 


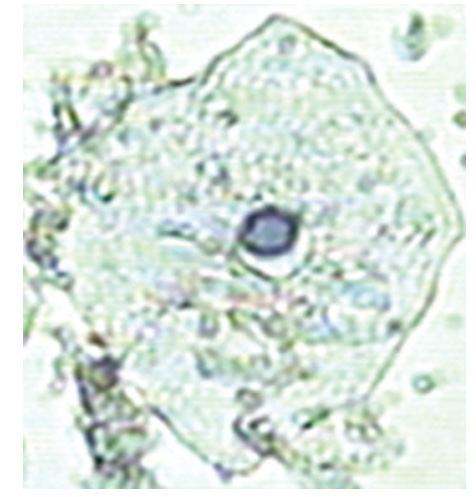

(a)

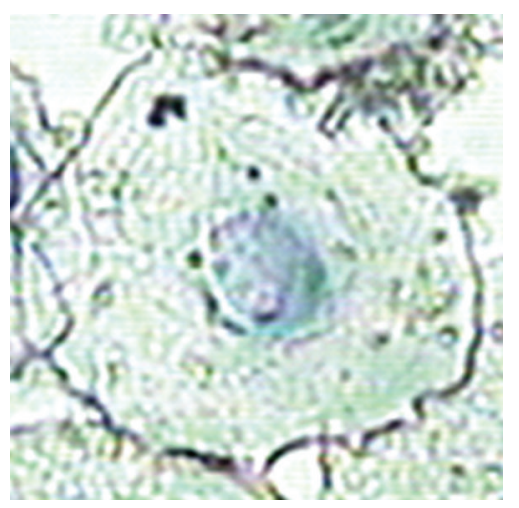

(b)

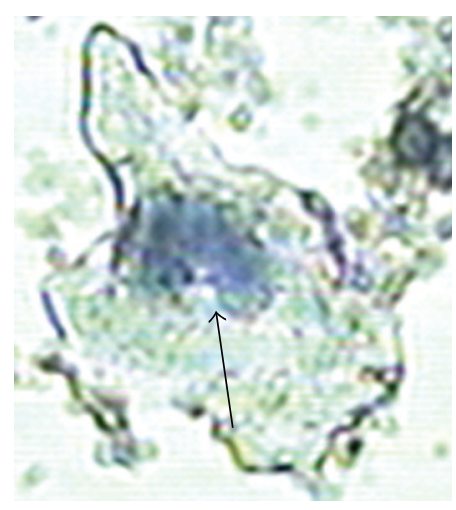

(c)

Figure 1: Morphology of cervical epithelial cells. Pap smear cells were obtained from patients and reviewed by a pathologist. From left to right, photos show a normal, LSIL, and HSIL cell. Arrow: the nucleus.

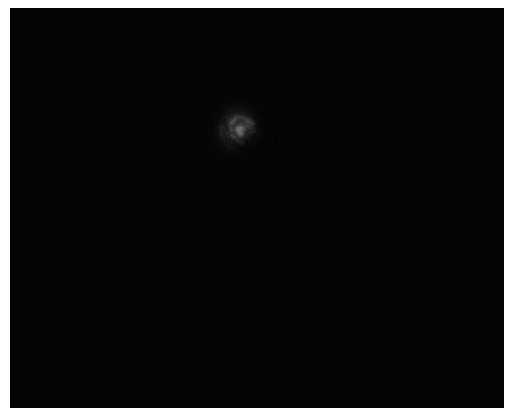

(a)

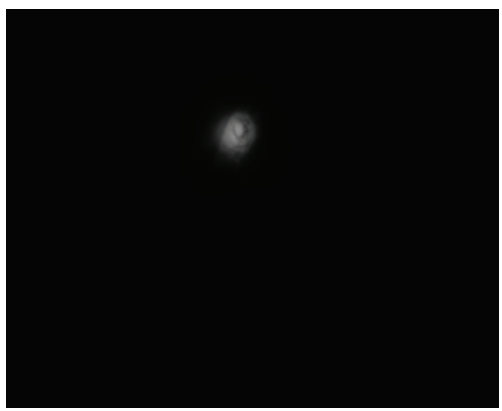

(b)

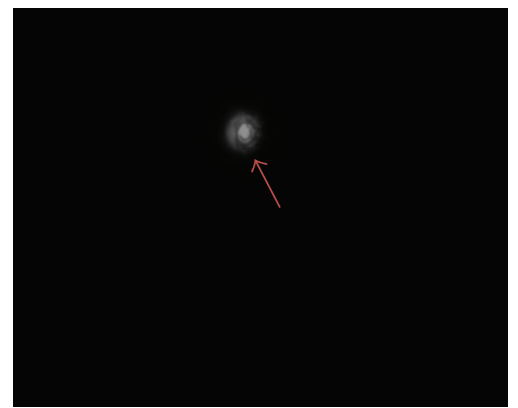

(c)

FIGURE 2: Images of cervical epithelial cells in liquid-based thin layer samples analyzed by CLASS microscopy. The photos show from left to right a normal, LSIL, and HSIL cell. Arrow: the nucleus. The diameter of the nuclei is larger than $2 \mu \mathrm{m}$.

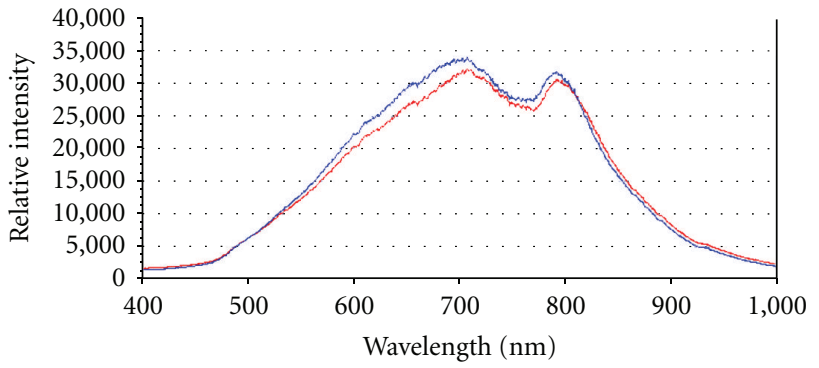

Figure 3: The light scattering curve as demonstrated by BWspec software analyses. Red color represented LSIL and blue represented HSIL.

3.3. Class Spectrum Analyses. Data collected from CLASS analyses of HSIL and non-HSIL cytology samples were then analyzed by BWspec spectral data acquisition software and plotted (Figure 3). Spectrum wavelengths $(\mathrm{nm})$ are represented on the abscissa, and the relative intensity is plotted on the ordinate scale. The data summarizes the spectra of the scattering light reflections from cell nuclei, which were normalized to the control and liquid-based normal cells.

As illustrated in Figure 3, the red curve shows the trend of back scattering lights of non-HSIL cells, and the blue curve represents the trend of back scattering lights of HSIL cells. Between 600 and $800 \mathrm{~nm}$ wavelengths, there were two peaks in the spectra curves, but the intensity differed in each. The HSIL cells (blue) trended toward a higher relative intensity, and the non-HSIL (red) trended relatively lower (Table 2). However, although HSIL cells trended higher in relative intensity, 4 of the 20 HSIL cases did not have higher intensity compared to the non-HSIL group. Conversely, 2 of the 20 cases of non-HSIL were higher in intensity compared to the HSIL group.

Comparing the trends of the HSIL and non-HSIL curves, the relative intensity of the HSIL cells was obviously higher than that of the non-HSIL at most wavelengths. There was a significant difference between intensities of the HSIL and non-HSIL at 600 and $800 \mathrm{~nm}$. However, there was no significant difference between them at wavelengths $>800 \mathrm{~nm}$. Sensitivity and specificity for HSIL and non-HSIL were $80 \%$ and $90 \%$, respectively, compared to confirmed diagnosis by cytology. 
TABLE 2: Relative intensity of HSIL and non-HSIL groups.

\begin{tabular}{lcccrc}
\hline & $600 \mathrm{~nm}$ & $650 \mathrm{~nm}$ & $700 \mathrm{~nm}$ & $750 \mathrm{~nm}$ & $800 \mathrm{~nm}$ \\
\hline Non-HSIL & $20714 \pm 210$ & $26911 \pm 326$ & $31413 \pm 168$ & $26632 \pm 292$ & $29409 \pm 221$ \\
HSIL & $21982 \pm 467$ & $29393 \pm 620$ & $33206 \pm 646$ & $27651 \pm 566$ & $30874 \pm 387$ \\
\hline
\end{tabular}

$P<0.05$, using Student's $t$-test.

\section{Discussion}

Cervical cancer, unlike most other cancers, is relatively easy to prevent and diagnose early, which contributes to reduced incidence and a better long-term survival rate. However, advanced stages of cervical cancers continue to be a serious health problem worldwide, especially in developing countries. Indeed, the incidence and mortality rates of cervical cancer are increasing in young women in China, although mortality for this cancer has significantly decreased in the United States and Western Europe. This may be due to the widespread use of the Pap smear screening test and safer sex practices. In an effort to improve the sensitivity and specificity of the Pap cytology test, we conducted the current study using CLASS technology. Our data demonstrates that CLASS microscopy was able to distinguish among normal, LSIL, and HSIL cells at specific wavelengths. CLASS microscopy is thus a useful and reliable technique for the detection of early cervical epithelial abnormality and could be used to help pathologists diagnose early cervical cancer.

Clinically it is crucial to distinguish precancerous lesions from normal and LSIL, because precancerous lesions could progress to invasive carcinoma if left untreated [9]. Many methods have been developed and used preclinically and clinically for the detection of early cervical cancer $[10,11]$. The goals of investigations of new techniques are to reduce the cost of screening, enable the practical surveillance of populations, or to improve the sensitivity to HSILs, which are considered cervical cancer precursors. Such investigations include the use of optical techniques.

Elastic light scattering spectroscopy can provide a valuable, noninvasive means to quantitatively probe tissue morphology and even detect biochemical changes in cells. However, despite growing evidence of the clinical utility of a variety of light scattering-based optical techniques, the biological bases of differences in scattering signals between normal and neoplastic tissues are not well understood.

The rationale for using optical techniques is to detect biochemical and morphological features that are concurrent with precancerous conditions. Light scattering is more sensitive to morphological changes or other currently used visualizing techniques. Therefore, this kind of noninvasive method has many advantages, including the ability to measure changes in nuclear size, which is one of the recognized morphological changes occurring during the transformation of normal cells to cancerous [9].

Some studies have demonstrated that the intensity of light scattering increases with progression of atypia [10]. The most dramatic differences in scattering occur between non-HSIL and HSIL cells because changes in nuclear size and DNA content are most pronounced between these two categories of cells. Studies have suggested that changes in scattering properties at high CIN grades are dominated by the effects of increased nuclear size and increased DNA content [11]. In fact, a complex spatial pattern is formed that is dependent on cell size, shape, refraction index, density, and morphology.

In this study, we used the optical method CLASS to distinguish HSIL of precancerous cervix cells from normal and LSIL samples in liquid-based specimens ex vivo. We found that CLASS was able to differentiate these in confirmed cytology samples, by the non-HSIL and HSILrelative intensity curves generated by the BWspec software. We showed that there was a significant difference in relative intensity between non-HSIL and HSIL cells in the 600$800 \mathrm{~nm}$ wavelength ranges. We could discriminate HSIL from non-HSIL cervical epithelial cells by differences in the curves, which represented the median light-scattering intensities of confirmed samples. Sixteen of the 20 HSIL cases demonstrated higher trends in relative intensity, and 18 of the 20 non-HSIL conformed to a lower trend in relative intensity. Therefore, in this study we established a novel model for diagnosis of liquid-based cervical cytological samples through the use of CLASS microscopy. As reported previously, this method is noninvasive with high sensitivity [12]. In addition, liquid-based specimens are easy to collect and preserve for analysis.

However, this study also has some limitations. For example, two of the LSIL cases showed high relative intensity. The reason may be due to overlapped or dividing cell nuclei. In addition, four cases of HSIL did not differ from the LSIL optimized curve. In the future, we will collect a larger number of samples to confirm our current data before translating this technique to the clinic.

\section{Conflict of Interests}

The authors have no conflict of interests.

\section{References}

[1] NCCC National Cervical Cancer Coalition, 2011, http://www .nccc-online.org/.

[2] E. P. Armstrong, "Prophylaxis of cervical cancer and related cervical disease: a review of the cost-effectiveness of vaccination against oncogenic HPV types," Journal of Managed Care Pharmacy, vol. 16, no. 3, pp. 217-230, 2010.

[3] K. Nanda, D. C. McCrory, E. R. Myers et al., "Accuracy of the papanicolaou test in screening for and follow-up of cervical cytologic abnormalities: a systematic review," Annals of Internal Medicine, vol. 132, no. 10, pp. 810-819, 2000. 
[4] L. A. Dainty, J. C. Elkas, G. S. Rose, and C. M. Zahn, "Controversial topics in abnormal cervical cytology: 'See and treat," Clinical Obstetrics and Gynecology, vol. 48, no. 1, pp. 193-201, 2005.

[5] H. Fang, L. Qiu, E. Vitkin et al., "Confocal light absorption and scattering spectroscopic microscopy," Applied Optics, vol. 46, no. 10, pp. 1760-1769, 2007.

[6] J. R. Mourant, T. J. Bocklage, T. M. Powers et al., "In vivo light scattering measurements for detection of precancerous conditions of the cervix," Gynecologic Oncology, vol. 105, no. 2, pp. 439-445, 2007.

[7] V. Backman, M. B. Wallace, L. T. Perelman et al., "Detection of preinvasive cancer cells," Nature, vol. 406, no. 6791, pp. 35-36, 2000.

[8] L. T. Perelman, V. Backman, M. Wallace et al., "Observation of periodic fine structure in reflectance from biological tissue: a new technique for measuring nuclear size distribution," Physical Review Letters, vol. 80, no. 3, pp. 627-630, 1998.

[9] J. R. Mourant, M. Canpolat, C. Brocker et al., "Light scattering from cells: the contribution of the nucleus and the effects of proliferative status," Journal of Biomedical Optics, vol. 5, no. 2, pp. 131-137, 2000.

[10] R. Drezek, M. Guillaud, T. Collier et al., "Light scattering from cervical cells throughout neoplastic progression: influence of nuclear morphology, DNA content, and chromatin texture," Journal of Biomedical Optics, vol. 8, no. 1, pp. 7-16, 2003.

[11] J. R. Mourant, J. P. Freyer, A. H. Hielscher, A. A. Eick, D. Shen, and T. M. Johnson, "Mechanisms of light scattering from biological cells relevant to noninvasive optical-tissue diagnostics," Applied Optics, vol. 37, no. 16, pp. 3586-3593, 1998.

[12] K. Kim, R. Zang, S. C. Choi, S. Y. Ryu, and W. K. Jae, "Current status of gynecological cancer in China," Journal of Gynecologic Oncology, vol. 20, no. 2, pp. 72-76, 2009. 

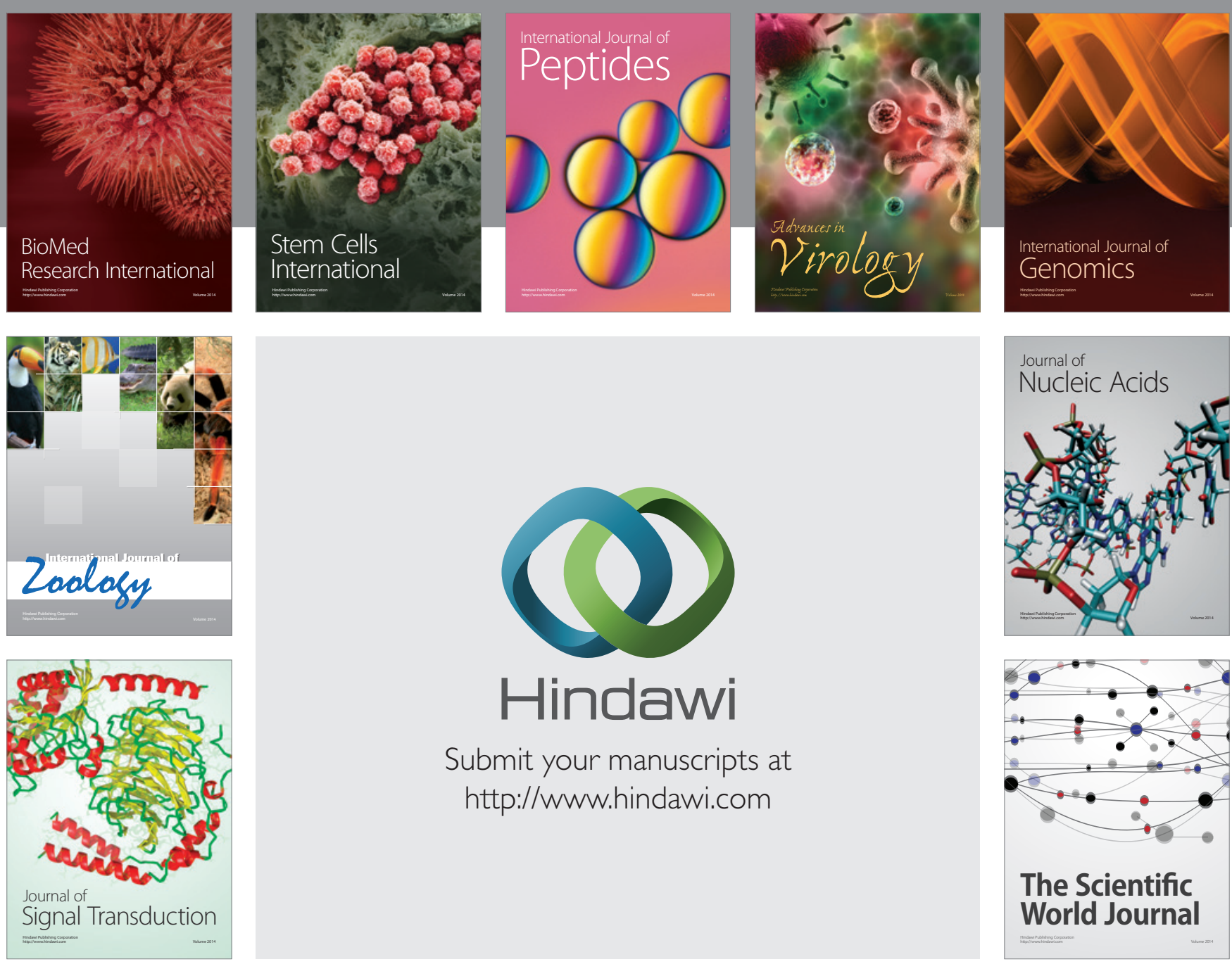

Submit your manuscripts at

http://www.hindawi.com
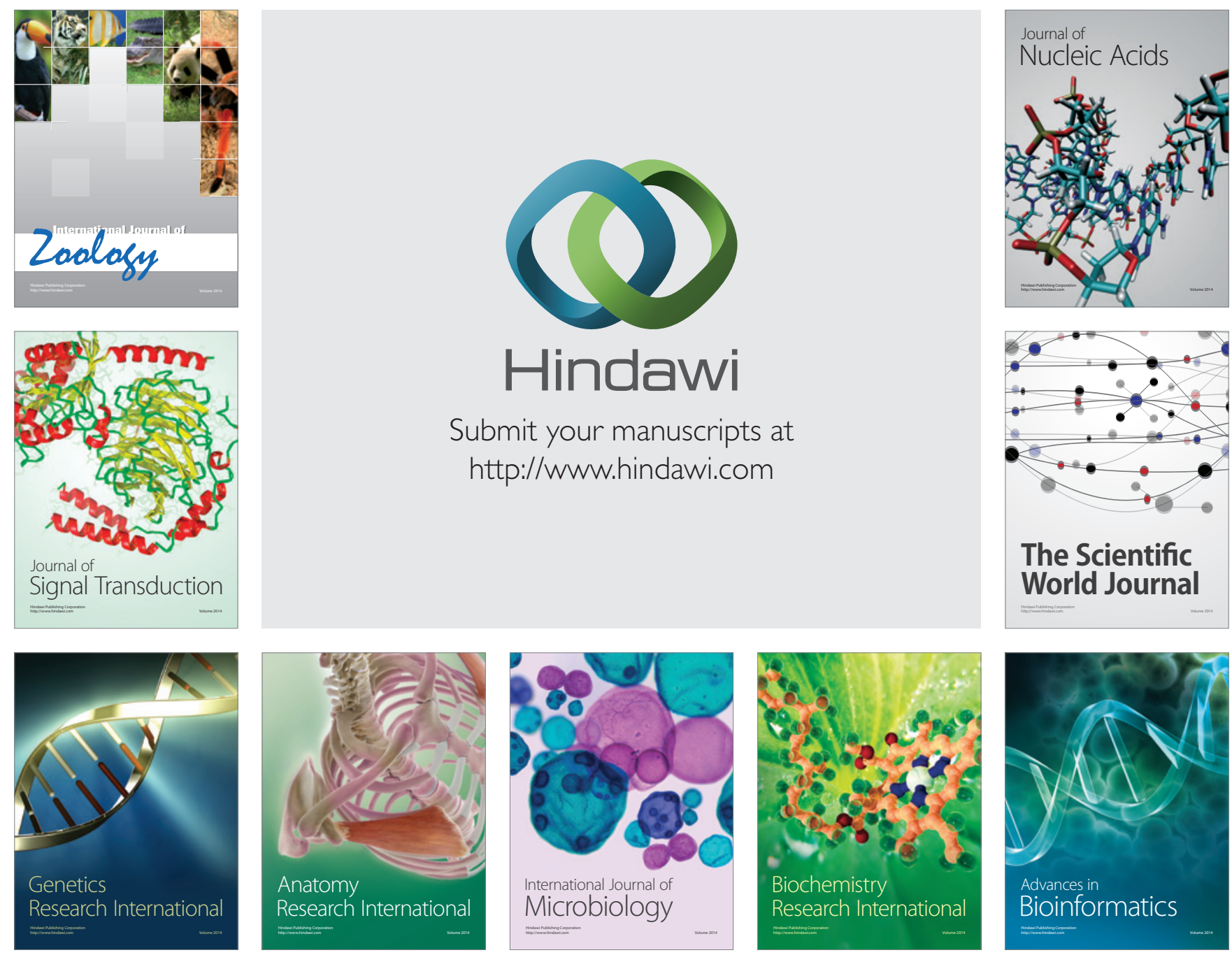

The Scientific World Journal
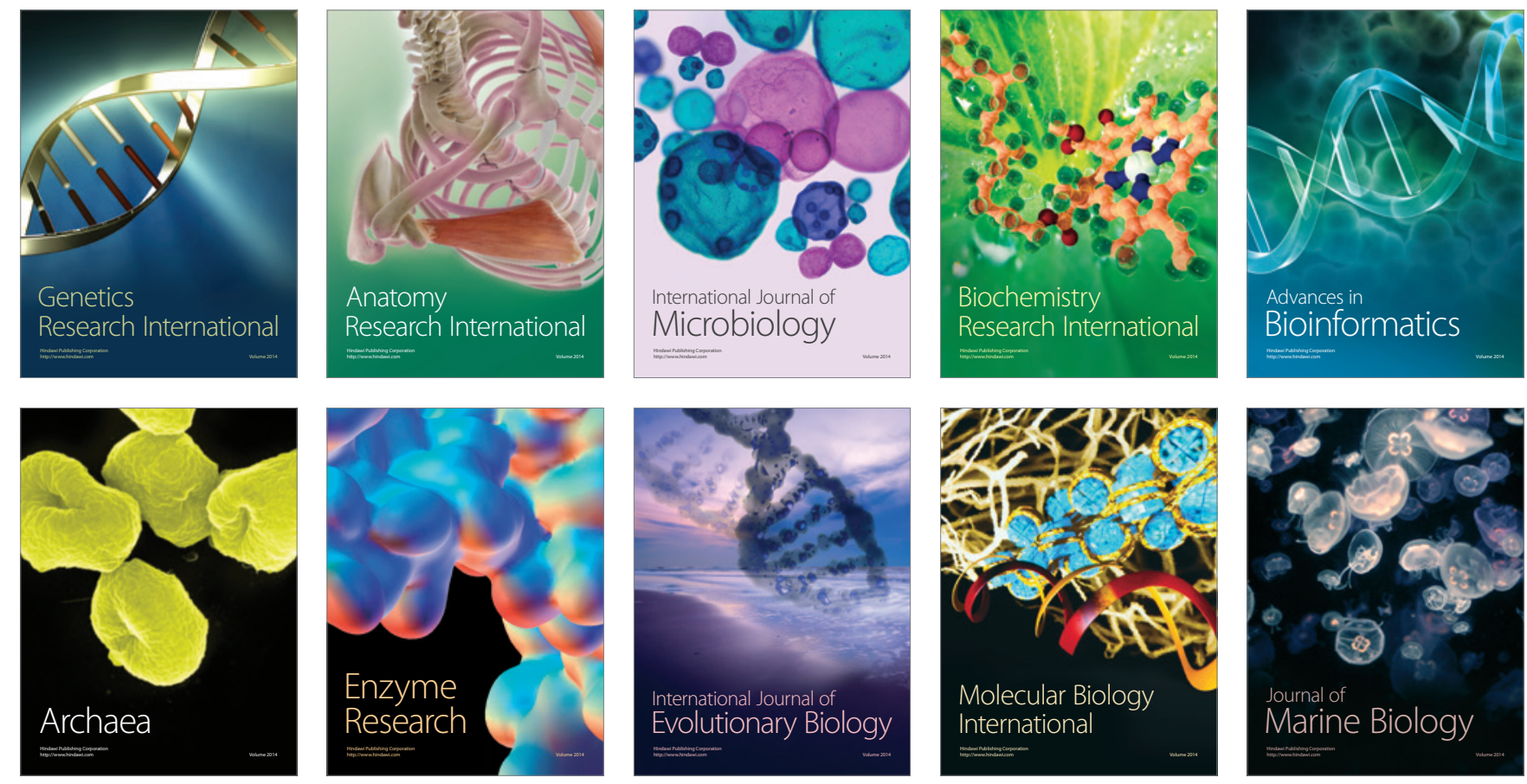